\title{
Biomarkers for predicting response to tyrosine kinase inhibitors in drug-sensitive and drug-resistant human bladder cancer cells
}

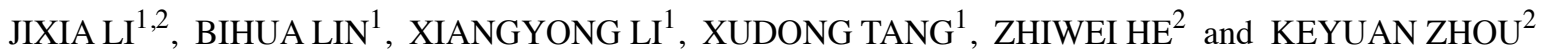 \\ ${ }^{1}$ Department of Biochemistry and Molecular Biology, and ${ }^{2}$ Key Laboratory for Medical Molecular Diagnostics \\ of Guangdong Province, Guangdong Medical College, Dongguan, Guangdong 523808, P.R. China
}

Received September 18, 2014; Accepted November 2, 2014

DOI: $10.3892 /$ or.2014.3639

\begin{abstract}
The epidermal growth factor receptor (EGFR) family is reportedly overexpressed in bladder cancer, and tyrosine kinase inhibitors (TKIs) have been suggested as treatment. Gefitinib (Iressa ${ }^{\circledR}$ ) is a selective inhibitor of the EGFR and lapatinib is a dual inhibitor of both the EGFR and HER2 (human EGFR type 2 receptor). Both compounds compete with the binding of ATP to the tyrosine kinase domain of the respective receptors to inhibit receptor autophosphorylation causing suppression of signal transduction. Unfortunately, resistance to these inhibitors is a major clinical issue. The purpose of the present study was to use protein array analysis to compare the signaling pathway(s) induced by gefitinib and lapatinib, in UM-UC-5 (drug-sensitive) and UM-UC-14 (drug-resistant) bladder cancer cells and to identify molecular markers that may be useful predictors of their efficacy. The results revealed that phosphorylation of EGFR, HER3, Met and ERK1/2 was markedly overexpressed in the sensitive cell line (UM-UC-5) and was strongly inhibited by the TKIs. Other notable differences included decreased phosphorylation of RSK, GSK3, AMPK, Akt and c-Jun by TKIs in the sensitive cells. In contrast, phosphorylated p53 was highly expressed in the resistant cell line (UM-UC-14) and TKIs had no effect in the resistant cells. Overall results suggest that phosphorylated HER3, ERK1/2 and p53 may be used as biomarkers to determine the sensitivity of bladder cancers to TKIs. In particular, a combination of these markers may be more likely to predict the sensitivity to TKIs.
\end{abstract}

\section{Introduction}

Bladder cancer is a common malignant disease in the USA and is the 4th most common cancer in men and the 10th in women (1). Genetic factors, including oncogenes, such as the epidermal growth factor receptor(EGFR) and tumor-suppressor genes, are risk factors for the development of bladder cancer.

Correspondence to: Professor Keyuan Zhou, Key Laboratory for Medical Molecular Diagnostics of Guangdong Province, Guangdong Medical College, Dongguan, Guangdong 523808, P.R. China

E-mail: 1983@gdmc.edu.cn; lijx313@vip.163.com

Key words: bladder cancer, Tyr-kinase inhibitor, gefitinib, lapatinib
Bladder cancer highly expresses EGFR and/or HER2 $(2,3)$. Transgene-driven overexpression of the EGFR within the bladder enhances tumor progression in mice, providing direct support for its importance in the biology of this disease (4). EGFR is a cell-surface receptor, belonging to the EGFR family of receptor tyrosine kinases. The EGFR family comprises four members: EGFR (HER1, ErbB1), ErbB2 (HER2), ErbB3 (HER3) and ErbB4 (HER4). The EGFR family members have four ectodomains, a single transmembrane domain and a cytoplasmic tail containing the active tyrosine kinase domain. Following the kinase domain, a C-terminal tail contains autophosphorylation sites that recruit signaling molecules (5). The EGFR-associated signaling pathway plays an important role in the development and progression of cancers. It has become one of the most important targets for anticancer drug discovery, and a large number of different small-molecule and antibodybased EGFR antagonists have been tested in clinical trials. Three small-molecule EGFR tyrosine kinase inhibitors (TKIs) are in clinical use and include gefitinib (ZD1839, Iressa $\left.{ }^{\circledR}\right)$, erlotinib (Tarceva ${ }^{\circledR}$ ) and lapatinib (GW 572016, Tykerb ${ }^{\circledR}$ ). All are based on a 4-anilinoquinazoline scaffold and target the ATP site to inhibit receptor autophosphorylation causing suppression of signal transduction. Gefitinib and erlotinib, selective EGFR inhibitors, target the active form of the kinase and have been approved for non-small cell lung cancer. Lapatinib, a dual inhibitor of EGFR and HER2, preferentially targets the inactive conformation and has been approved for HER2positive breast cancer $(6,7)$. Although TKIs display a survival advantage in clinical trials, only a minority of patients appear to respond to this approach. Resistance to TKIs has become a major clinical issue. Thus, research is needed to identify and validate predictive factors that can be used to select patients with disease likely to respond to TKIs. Recent data showed that the tumor response is not associated with a higher proportion of EGFR-positive tumor cells or more intensive EGFR staining in lung cancer (8). Therefore, the identification of additional predictive markers is extremely important.

In the present study, we compared the signaling pathway(s) induced by TKIs (gefitinib and lapatinib), in UM-UC-5 (drugsensitive) and UM-UC-14 (drug-resistant) bladder cancer cell lines and identified molecular markers as predictors of their efficacy. Here, we report that HER3 and ERK1/2 phosphorylation was extremely high in the UM-UC-5 cells and substantially suppressed by TKIs. However, a phosphorylated mutant p53 

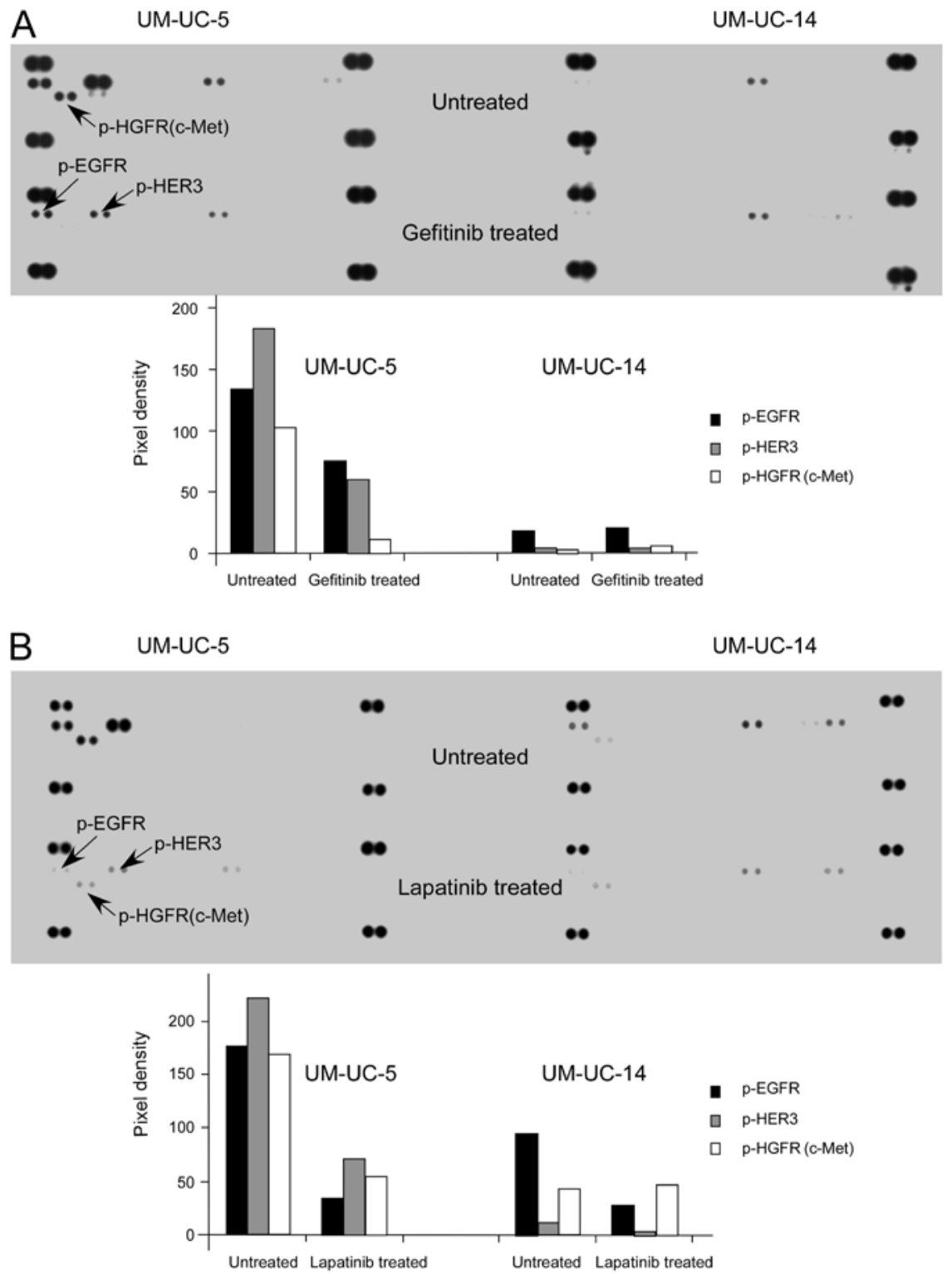

Figure 1. UM-UC-5 cells are sensitive to TKIs, and show overexpression of phosphorylated EGFR, HER3 and MET. (A) Results of the phospho-RTK protein array analysis using UM-UC-5 and UM-UC-14 cells treated or not treated with gefitinib. (B) Results of the phospho-RTK protein array analysis using UM-UC-5 and UM-UC-14 cells treated or not treated with lapatinib. For A and B, both cell lines were treated with $2.5 \mu \mathrm{M}$ gefitinib or lapatinib for $24 \mathrm{~h}$, and the cell lysates were hybridized to the phospho-RTK array. In the array, each RTK is spotted in duplicate. Hybridization signals at the corners serve as controls. TKIs, tyrosine kinase inhibitors; EGFR, epidermal growth factor receptor; RTK receptor tyrosine kinase.

was overexpressed in the UM-UC-14 cells. These data suggest that increased activation of HER 3 and ERK1/2 may be related to the response to TKIs, and that enhanced phosphorylation of mutant p53 may be linked with resistance to TKIs.

\section{Materials and methods}

Reagents. Eagle's minimum essential medium (MEM) was purchased from Invitrogen (Carlsbad, CA, USA). Fetal bovine serum (FBS) was purchased from Gemini Bio-Products (Calabasas, CA, USA) and the antibiotics (penicillin and streptomycin) were from Invitrogen. The human phosphoRTK array kit, human phospho-MAPK array kit and human phospho-kinase array kit were purchased from R\&D Systems (Minneapolis, MN, USA). The antibodies against phosphorylated HER3 (Tyr1289), phosphorylated ERKs
(Tyr-202/Tyr-204) and phosphorylated p53 (Ser15) were purchased from Cell Signaling Biotechnology (Beverly, MA, USA). The protein assay kit was from Bio-Rad (Hercules, CA, USA).

Cell culture. The bladder cancer UM-UC-5 and UM-UC-14 cells were cultured in monolayers at $37^{\circ} \mathrm{C}$ in a $5 \% \mathrm{CO}_{2}$ incubator in MEM containing 10\% FBS and penicillin/streptomycin.

Protein array. Each cell line was cultured to $90 \%$ confluency and then starved $24 \mathrm{~h}$ in serum-free media. They were treated, respectively, with $2.5 \mu \mathrm{M}$ gefitinib or lapatinib in culture medium containing $10 \%$ FBS for $24 \mathrm{~h}$ and then harvested. Cell samples were disrupted and then proteins were extracted. The protein concentration was determined using a dye-binding protein assay kit (Bio-Rad) as described in the manufacturer's 

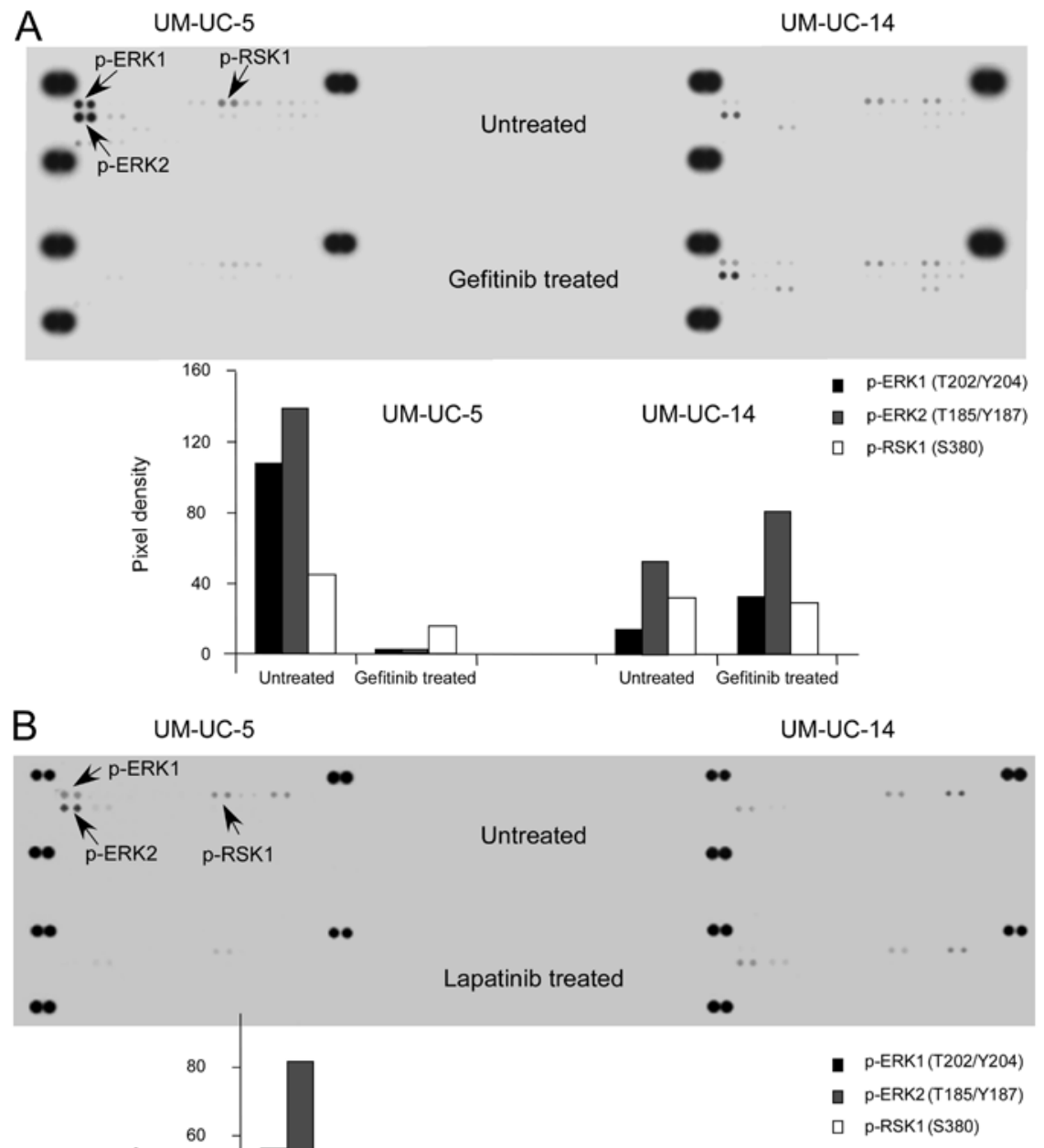
Figure 2. UM-UC-5 cells, which are sensitive to TKIs, overexpress phosphorylated ERK1 and ERK2. (A) The results of the phospho-MAPK protein array UM-UC-5 and UM-UC-14 cells treated or not treated with lapatinib. For A and B, both cell lines were treated with $2.5 \mu \mathrm{M}$ gefitinib or lapatinib for $24 \mathrm{~h}$, and the cell lysates were hybridized to the phospho-MAPK protein array. In the array, each MAPK is spotted in duplicate. Hybridization signals at the corners serve as controls TKIs, tyrosine kinase inhibitors.

manual. Following the instructions provided with the protein arrays, cell lysates were subjected to Proteome Profiler ${ }^{\mathrm{TM}}$ Arrays including tyrosine phosphorylation of receptor tyrosine kinases (RTKs) (42 signals), phosphorylation of mitogen-activated protein kinases (MAPKs) (21 signals) and phospho-kinase (46 signals) array analysis.

Western blot analysis. After cells $\left(1 \times 10^{6}\right)$ were cultured in a $10-\mathrm{cm}$ dish overnight, they were starved in serum-free medium for another $24 \mathrm{~h}$ to eliminate the influence of FBS on the activation of mitogen-activated protein kinases. The cells were then treated with $2.5 \mu \mathrm{M}$ gefitinib or lapatinib for $24 \mathrm{~h}$ in culture medium containing $10 \%$ FBS. The harvested cells were disrupted, and the supernatant fractions were boiled for $5 \mathrm{~min}$. The protein concentration was determined using a dye-binding protein assay kit (Bio-Rad). Lysate proteins
(50 $\mu \mathrm{g})$ were subjected to 8-10\% SDS-PAGE and electrophoretically transferred to a polyvinylidene difluoride membrane (GE Healthcare). After blotting, the membrane was incubated with a specific primary antibody at $4^{\circ} \mathrm{C}$ overnight. Protein bands were visualized using a chemiluminescence detection kit (GE Healthcare) after hybridization with an AP-linked secondary antibody.

\section{Results}

Phosphorylated HER3 is highly expressed in the drug-sensitive bladder cancer cells and is substantially decreased by TKIs. The EGFR family plays a central role in the pathogenesis and progression of bladder cancer (9). Manifold actions for other growth factor receptors, e.g., MET, have been reported in cancer (10). MET encodes a transmembrane tyrosine kinase 
A

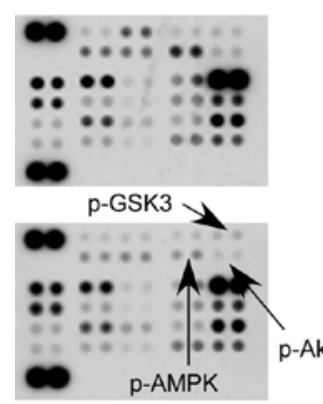

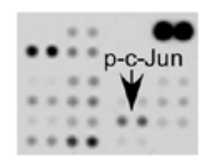

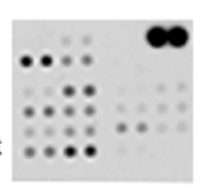

Untreated

Gefitinib treated
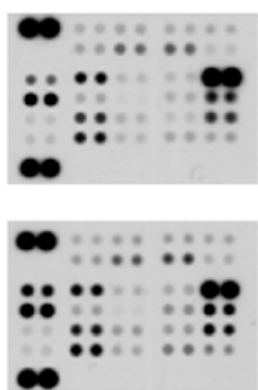

UM-UC-14
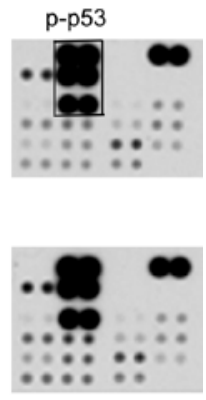

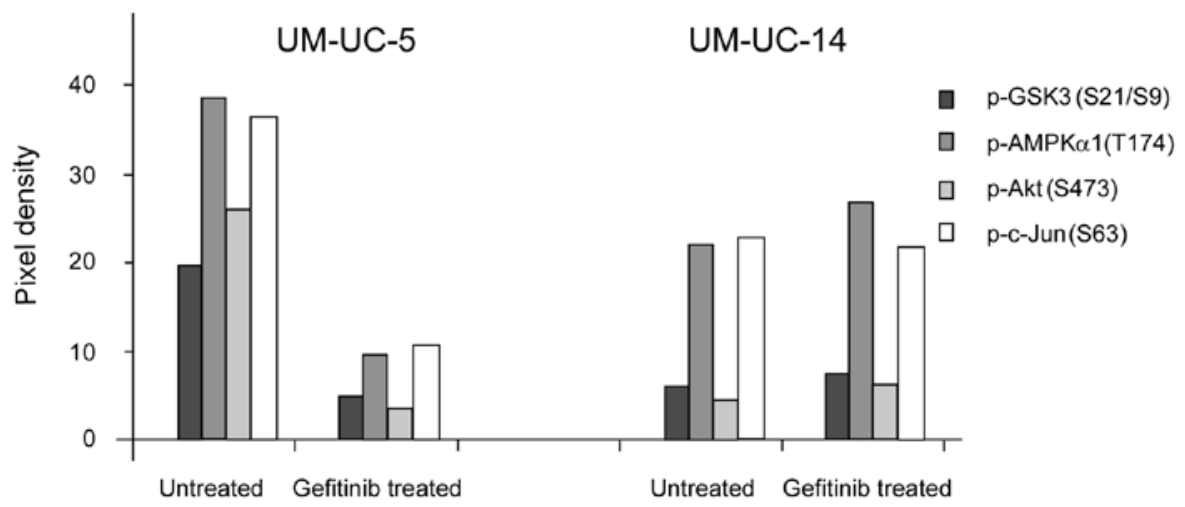

UM-UC-5 UM-UC-14

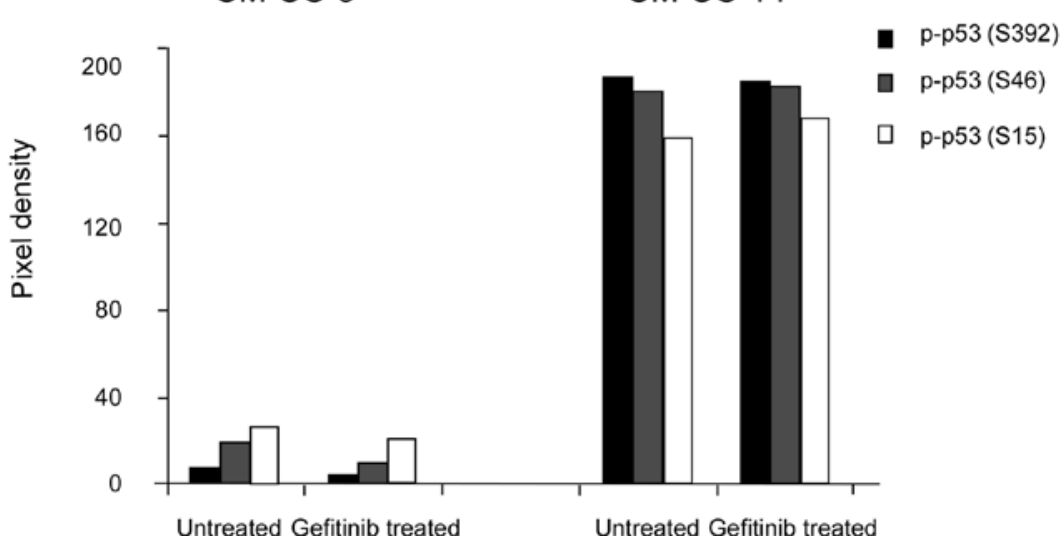

Figure 3. UM-UC-14 cells, which are resistant to TKIs, express high levels of phosphorylated mutant p53. (A) The results of the phospho-kinase protein array analysis using UM-UC-5 and UM-UC-14 cells treated or not treated with gefitinib. Both cell lines were treated with $2.5 \mu \mathrm{M}$ gefitinib for $24 \mathrm{~h}$, and the cell lysates were hybridized to the phospho-kinase array. In the array, each kinase is spotted in duplicate. Hybridization signals at the corners serve as control. TKIs, tyrosine kinase inhibitors.

receptor for the hepatocyte growth factor (scatter factor), and MET amplification has been correlated with resistance to TKIs in lung cancer (10). We first examined the relative level of tyrosine phosphorylation of RTKs in both bladder cancer cell lines (UM-UC-5 and UM-UC-14) before and after treatment with TKIs (gefitinib or lapatinib). The results revealed that phosphorylated EGFR, HER3 and MET were overexpressed in the sensitive cell line (UM-UC-5), yet were barely detectable in the resistant cell line (UM-UC-14; Fig. 1A and B). Gefitinib and lapatinib markedly suppressed EGFR, HER3 and MET phosphorylation in the UM-UC-5 cells, yet had no effect in the UM-UC-14 cells. Based on these data, the most notable difference between these two cell lines was the HER3 phosphorylation and the effectiveness of TKIs in suppressing this signal in UM-UC-5 cells.

Phosphorylated ERK1/2 is overexpressed in sensitive bladder cancer cells and is substantially inhibited by TKIs. Next, we investigated the level of phosphorylation of the MAPK pathways induced by gefitinib and lapatinib in the UM-UC-5 and UM-UC-14 cell lines. The results revealed that the level of ERK1/2 phosphorylation was higher in the UM-UC-5 cell line when compared with that in the UM-UC-14 cells (Fig. 2A and B). The TKIs markedly suppressed ERK1/2 
B

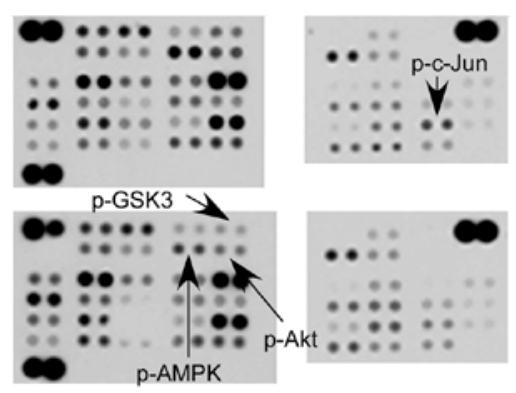

UM-UC-14

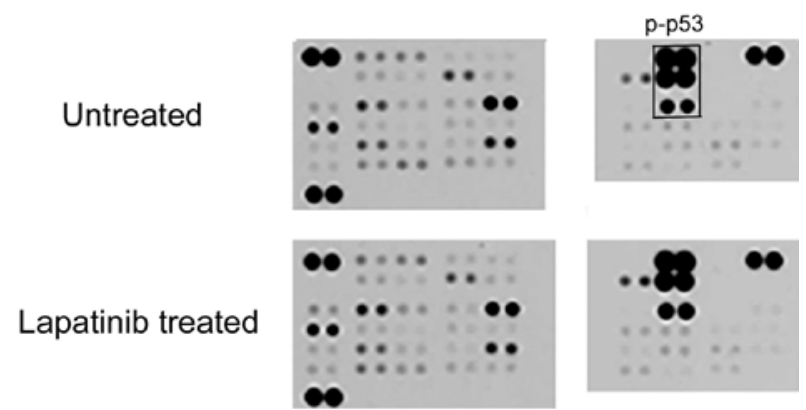

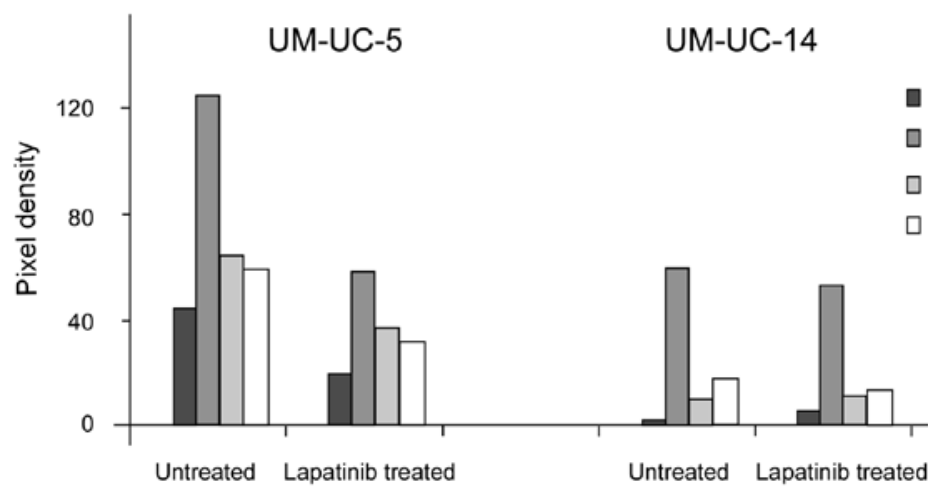

p-GSK3 (S21/S9)

口 $\mathrm{p}-\mathrm{AMPK} \alpha 1$ (T174)

口 p-Akt (\$473)

( p-c-Jun (S63)

UM-UC-5

UM-UC-14

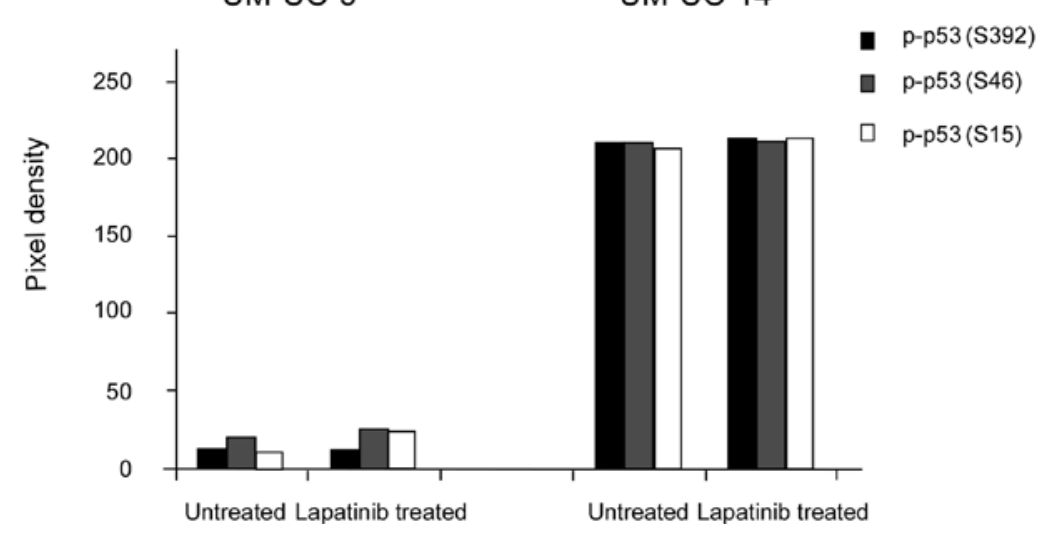

Figure 3. Continued. (B) The results of the phospho-kinase protein array analysis using UM-UC-5 and UM-UC-14 cells treated or not treated with lapatinib. Both cell lines were treated with $2.5 \mu \mathrm{M}$ lapatinib for $24 \mathrm{~h}$, and the cell lysates were hybridized to the phospho-kinase array. In the array, each kinase is spotted in duplicate. Hybridization signals at the corners serve as control. TKIs, tyrosine kinase inhibitors.

phosphorylation in the UM-UC-5 cells, yet had no effect on the UM-UC-14 cells. Phosphorylated RSK was also decreased in the sensitive cells following drug treatment. However, the most important event was that phosphorylation of ERK1/2, which is upstream of RSK and was highly expressed in the sensitive cells, was substantially decreased by the TKIs.

TKI-resistant bladder cancer cells overexpress a mutant phosphorylated p53. The activation of the EGFR turns on several different signaling pathways, including MAPKs, PI3K/Akt, STAT and SRC/FAK (11). We found that the phosphorylation of GSK3, AMPK, Akt and c-Jun was higher in the UM-UC-5 cells and was suppressed by TKIs, whereas these proteins were not affected in the UM-UC-14 cells (Fig. 3A and B). Notably, phosphorylated p53 was markedly overexpressed in the untreated resistant cells compared with that in the sensitive cells (Fig. 3A and B). As for the other signals, no difference was observed in either cell line.

In order to confirm the protein array data, we performed western blot analysis to examine the phosphorylation of HER3, ERK1/2 and p53 in these two cell lines both before and after TKI treatments. Western blot results also showed that phosphorylated HER3 and ERK1/2 were overexpressed in the UM-UC-5 cells and were strongly suppressed following gefitinib or lapatinib treatment and that phosphorylated p53 was highly expressed in the UM-UC-14 cells (Fig. 4). 


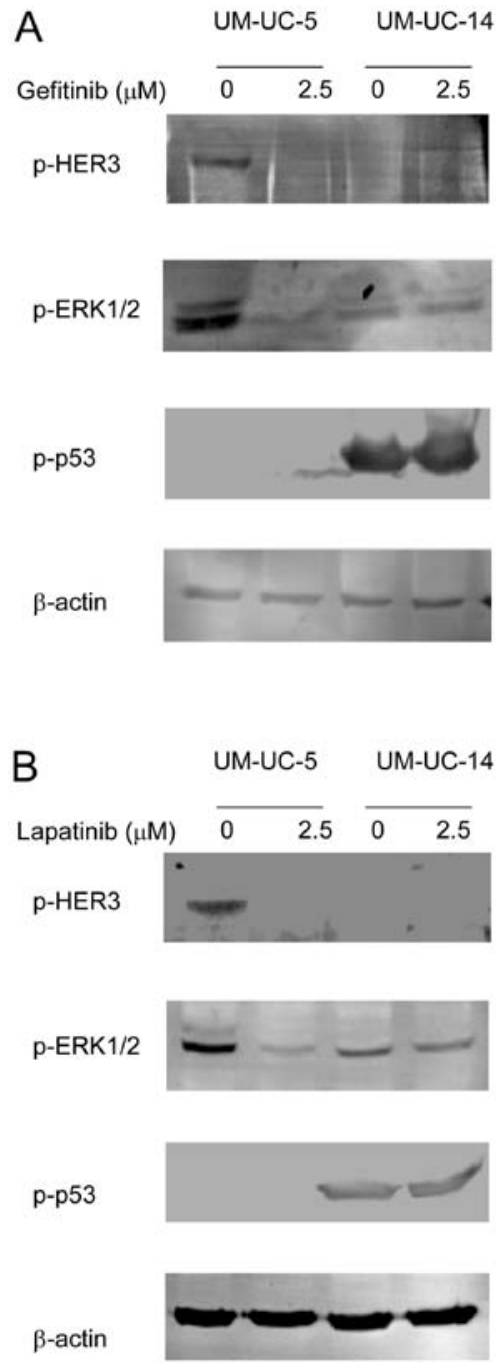

Figure 4. Verification of the array data by western blot analysis. (A) Effect of gefitinib on the phosphorylation of HER3, ERK1/2 and p53 in the UM-UC-5 and UM-UC-14 cells. (B) Effect of lapatinib on the phosphorylation of HER3 ERK1/2 and $\mathrm{p} 53$ in the UM-UC-5 and UM-UC-14 cells. Both cell lines were treated with $2.5 \mu \mathrm{M}$ gefitinib or lapatinib for $24 \mathrm{~h}$, and the cell lysates were subjected to immunoblotting to detect the indicated proteins.

\section{Discussion}

EGFR mutation and amplification and dysfunction of other members of the EGFR family and downstream of EGFR signaling are likely involved in the response of lung cancer to TKI treatment (12). Previous findings from a phase II study of erlotinib in patients with NSCLC did not show a correlation between the degree of EGFR expression and response to therapy, and suggest that alternative markers (indicative of activation of the receptor or the pathway, or both) are more likely to be predictive of sensitivity to EGFR inhibition (8). EGFR and/or HER2 are reported to be deregulated in bladder cancer, yet mutations within the EGFR and expression of EGFR vIII are rare events in bladder cancer (13). Recent findings revealed that although EGFR levels appear to roughly correspond with responsiveness, they cannot be used alone to identify bladder cancers that will be sensitive to TKIs (4). EGFR and/or HER2 are associated with poor prognosis (14), whereas HER3 and/or HER4 are related to a better outcome for bladder cancer patients (15). Expression of EGFR and HER2 alone does not appear to be associated with survival. However, high expression of EGFR along with high expression of HER3 and/or HER4 was found to correspond to a better prognosis compared with high expression of EGFR together with low expression of HER3 and/or HER4 (16). Therefore, the prognostic significance of EGFR and/or HER2 overexpression is modulated by expression of HER3 and/or HER4, indicating the complexity of interactions between the different EGFRs. The present study demonstrated that the phosphorylated levels of EGFR and particularly, HER3, were high whereas phosphorylation of HER2 and HER4 was not observed in the TKI-sensitive bladder cancer cells. Following TKI treatment, phosphorylation of EGFR and HER3 was markedly decreased in the sensitive cells and no change was observed in the resistant cells. Additional evidence suggests that the functions of HER3 may be important in the prediction of sensitivity to TKIs (17). For example, in a comparison of lung cancer cell lines that are sensitive or resistant to gefitinib, the best marker of sensitivity to gefitinib was found to be the sensitivity of HER3 signaling (18). In agreement with previous data, an increased activation of HER 3 was also found to be related to the response of bladder cancer to TKIs.

EGFR and HER2 are classically coupled to the Ras/Raf/ MEK/ERK-dependent pathway, whereas HER3 is a potent activator of PI3K/Akt (19). Studies have shown that a majority of bladder cancers express activated Ras, and that this oncogenic activation is an important tumorigenic factor (20-23). Phosphorylated ERK is the key downstream target of the Ras/Raf/MEK/ERK signaling pathway, and deregulation of this pathway occurs in approximately one-third of all human cancers (24). Furthermore, phosphorylated ERK1/2 may be a potential predictive marker of sensitivity to sorafenib in hepatocellular carcinoma. Sorafenib was reported to inhibit ERK1/2 phosphorylation and was dependent on the degree of basal expression level of phosphorylated ERK1/2 (25). We report a similar finding in that TKI-sensitive bladder cancer cells exhibited a high expression level of phosphorylated ERK $1 / 2$ and the elevated phosphorylation was suppressed by TKIs. Hence, phosphorylated ERK1/2 may be an important biomarker for the prediction of sensitivity to TKIs in bladder cancer.

Bladder cancers exhibit frequent alterations in the tumorsuppressor gene p53, and mutant p53 expression demonstrates a strong association with disease grade and stage, as well as with survival (26). Aberrations of p53 are usually associated with drug resistance. Recent data showed that human urothelial cells with loss of p53 function displayed reduced sensitivity to TKIs (27) and p53 is required for maximal sensitivity to gefitinib-induced apoptosis in non-small cell lung cancer (28). One point mutation (codon 135, cysteine to serine) was identified in the UM-UC-14 cells (data not shown). Our data showed that phosphorylated mutant p53 was highly expressed in the resistant bladder cancer cells. p53 cysteine substitution at C135 was found to decrease human p53 activity in wild-type yeast (29), thus silencing of mutant p53 had no effect on the sensitivity to TKIs (data not shown). These results suggest that overexpressed phosphorylated p53 may be a marker of resistance to TKIs in bladder cancer. 
In conclusion, phosphorylated HER3, ERK1/2 and p53 may be used as biomarkers to determine bladder cancer sensitivity to TKIs. A combination of these markers may be more likely to predict sensitivity to TKIs.

\section{Acknowledgements}

This study was supported by grants from the National Natural Science Foundation of China (nos. 81201710 and 81272434), the Guangdong Natural Science Foundation (S2012010008259), and the Guangdong Medical College research grant (XG 1101 and STIF 201105).

\section{References}

1. van Rhijn BW, Burger M, Lotan Y, et al: Recurrence and progression of disease in non-muscle-invasive bladder cancer: from epidemiology to treatment strategy. Eur Urol 56: 430-442, 2009.

2. McHugh LA, Sayan AE, Mejlvang J, et al: Lapatinib, a dual inhibitor of ErbB-1/-2 receptors, enhances effects of combination chemotherapy in bladder cancer cells. Int J Oncol 34: 1155-1163, 2009.

3. Wang X, Zhang S, MacLennan GT, et al: Epidermal growth factor receptor protein expression and gene amplification in small cell carcinoma of the urinary bladder. Clin Cancer Res 13: 953-957, 2007.

4. Shrader M, Pino MS, Brown G, et al: Molecular correlates of gefitinib responsiveness in human bladder cancer cells. Mol Cancer Ther 6: 277-285, 2007.

5. Johnson LN: Protein kinase inhibitors: contributions from structure to clinical compounds. Q Rev Biophys 42: 1-40, 2009.

6. McHugh LA, Kriajevska M, Mellon JK and Griffiths TR Combined treatment of bladder cancer cell lines with lapatinib and varying chemotherapy regimens - evidence of scheduledependent synergy. Urology 69: 390-394, 2007.

7. Shrader M1, Pino MS, Lashinger L, et al: Gefitinib reverses TRAIL resistance in human bladder cancer cell lines via inhibition of AKT-mediated X-linked inhibitor of apoptosis protein expression. Cancer Res 67: 1430-1435, 2007.

8. Dancey JE and Freidlin B: Targeting epidermal growth factor receptor - are we missing the mark? Lancet 362: 62-64, 2003

9. Xu L, Hausmann M, Dietmaier W, et al: Expression of growth factor receptors and targeting of EGFR in cholangiocarcinoma cell lines. BMC Cancer 10: 302, 2010.

10. Engelman JA, Zejnullahu K, Mitsudomi T, et al: MET amplification leads to gefitinib resistance in lung cancer by activating ERBB3 signaling. Science 316: 1039-1043, 2007.

11. Laurent-Puig P, Lievre A and Blons H: Mutations and response to epidermal growth factor receptor inhibitors. Clin Cancer Res 15: 1133-1139, 2009.

12. Shigematsu H and Gazdar AF: Somatic mutations of epidermal growth factor receptor signaling pathway in lung cancers. Int J Cancer 118: 257-262, 2006.
13. Villares GJ, Zigler M, Blehm K, et al: Targeting EGFR in bladder cancer. World J Urol 25: 573-579, 2007.

14. Memon AA, Sorensen SB and Nexo E: The epidermal growth factor family has a dual role in deciding the fate of cancer cells. Scand J Clin Lab Invest 66: 623-630, 2006.

15. Memon AA, Sorensen BS, Melgard P, et al: Expression of HER3, HER4 and their ligand heregulin-4 is associated with better survival in bladder cancer patients. Br J Cancer 91: 2034-2041, 2004.

16. Memon AA, Sorensen BS, Meldgaard P, et al: The relation between survival and expression of HER1 and HER2 depends on the expression of HER3 and HER4: a study in bladder cancer patients. Br J Cancer 94: 1703-1709, 2006.

17. Amin DN, Campbell MR and Moasser MM: The role of HER3, the unpretentious member of the HER family, in cancer biology and cancer therapeutics. Semin Cell Dev Biol 21: 944-950, 2010.

18. Engelman JA, Jänne PA, Mermel C, et al: ErbB-3 mediates phosphoinositide 3-kinase activity in gefitinib-sensitive non-small cell lung cancer cell lines. Proc Natl Acad Sci USA 102: 3788-3793, 2005.

19. Koutras AK, Fountzilas G, Kalogeras KT, et al: The upgraded role of HER 3 and HER4 receptors in breast cancer. Crit Rev Oncol Hematol 74: 73-78, 2010.

20. Fontana D, Bellina M, Scoffone C, et al: Evaluation of c-ras oncogene product (p21) in superficial bladder cancer. Eur Urol 29: 470-476, 1996.

21. Przybojewska B, Jagiello A and Jalmuzna P: $H-R A S, K-R A S$, and $N-R A S$ gene activation in human bladder cancers. Cancer Genet Cytogenet 121: 73-77, 2000.

22. Rose A, Grandoch M, vom Dorp F, et al: Stimulatory effects of the multi-kinase inhibitor sorafenib on human bladder cancer cells. Br J Pharmacol 160: 1690-1698, 2010.

23. Vageli D, Kiaris H, Delakas D, Anezinis P, Cranidis A and Spandidos DA: Transcriptional activation of $\mathrm{H}-$ ras, K-ras and N-ras proto-oncogenes in human bladder tumors. Cancer Lett 107: 241-247, 1996.

24. Dhillon AS, Hagan S, Rath O and Kolch W: MAP kinase signalling pathways in cancer. Oncogene 26: 3279-3290, 2007.

25. Zhang Z, Zhou X, Shen H, et al: Phosphorylated ERK is a potential predictor of sensitivity to sorafenib when treating hepatocellular carcinoma: evidence from an in vitro study. BMC Med 7: 41, 2009.

26. Proctor I, Stoeber K and Williams GH: Biomarkers in bladder cancer. Histopathology 57: 1-13, 2010.

27. MacLaine NJ, Wood MD, Holder JC, et al: Sensitivity of normal, paramalignant, and malignant human urothelial cells to inhibitors of the epidermal growth factor receptor signaling pathway. Mol Cancer Res 6: 53-63, 2008.

28. Rho JK, Choi YJ, Ryoo BY, et al: p53 enhances gefitinibinduced growth inhibition and apoptosis by regulation of Fas in non-small cell lung cancer. Cancer Res 67: 1163-1169, 2007.

29. Stoner CS, Pearson GD, Koc A, et al: Effect of thioredoxin deletion and p53 cysteine replacement on human p53 activity in wild-type and thioredoxin reductase null yeast. Biochemistry 48 : 9156-9169, 2009. 